\title{
A somatic TSHR mutation in a patient with lung adenocarcinoma with bronchioloalveolar carcinoma, coronary artery disease and severe chronic obstructive pulmonary disease
}

\author{
JAMES WAN SOO KIM ${ }^{1}$, SHARON LEE ${ }^{1}$, NATALIE LUI ${ }^{1}$, HELEN CHOI ${ }^{1,2}$, MICHAEL MULVIHILL ${ }^{1}$, \\ LI TAI FANG ${ }^{1}$, HIO CHUNG KANG ${ }^{2}$, YONG-WON KWON ${ }^{3}$, DAVID JABLONS ${ }^{1,2}$ and IL-JIN KIM ${ }^{1,2}$
}

${ }^{1}$ Thoracic Oncology Laboratory, Department of Surgery, ${ }^{2}$ Comprehensive Cancer Center, University of California San Francisco, San Francisco, CA; ${ }^{3}$ Life Sciences Division, Lawrence Berkeley National Laboratory (LBNL), Berkeley, CA, USA

Received April 4, 2012; Accepted May 14, 2012

DOI: 10.3892/or.2012.1938

\begin{abstract}
In a screen for thoracic malignancy-associated markers, thyroid stimulating hormone receptor (TSHR) was identified as a candidate as it binds to the previouslycharacterized lung cancer marker NKX2-1. We screened for mutations in all coding regions of the TSHR gene in 96 lung adenocarcinoma samples and their matched adjacent normal lung samples. We found one patient with a somatic mutation at codon 458 (exon 10), which is located at the transmembrane domain where most TSHR mutations have been found in thyroid-related diseases. This patient had lung adenocarcinoma with BAC (bronchioloalveolar carcinoma) features in the setting of a prior medical history significant for carotid stenosis and severe chronic obstructive pulmonary disease (COPD). In order to characterize the genetic features of TSHR in lung cancer, we checked for TSHR expression and copy number in the 96 lung cancer tissues. TSHR protein expression was generally overexpressed in multiple thoracic malignancies (adenocarcinoma, squamous cell carcinoma and malignant pleural mesothelioma) by immunohistochemistry. Our data suggest that aberrant TSHR function may contribute to lung cancer development or a subgroup of lung cancer with specific clinical phenotypes.
\end{abstract}

\section{Introduction}

Lung cancer is one of the worst prognostic human cancers, taking more lives than any other human cancer worldwide (1). Progress in therapy for lung cancer has made recent progress

Correspondence to: Dr Il-Jin Kim or Dr David Jablons, Department of Surgery, University of California San Francisco, 2340 Sutter Street, Room N225, San Francisco, CA 94115, USA

E-mail: kimij@cc.ucsf.edu

E-mail: david.jablons@ucsfmedctr.org

Key words: thyroid stimulating hormone receptor, lung cancer, adenocarcinoma, mutation with the identification of molecular markers such as EGFR and EML4-ALK (2-4). Gefitinib (Tarceva) targeting EGFR and Crizotinib (Xalkori) targeting EML4-ALK are leading examples of molecular targeting in lung cancer therapeutics $(2,4)$. Patients with EGFR mutations have responded well to Tarceva. However, many patients later relapse due to a secondary EGFR mutation (T790M), decreasing the advantage of Tarceva treatment with respect to patient prognosis and survival (5). In 2007, a new fusion protein, $E M L 4-A L K$, was identified in a Japanese lung cancer patient without EGFR or K-ras mutations (3). The incidence of this fusion protein is greater in Asian patients without EGFR or K-ras mutations (6). Encouragingly, patients with EML4-ALK showed a dramatic response to an ALK inhibitor (7) and in 2011, the FDA approved Crizotinib for the treatment of non-small-cell lung cancer (NSCLC) in patients with EML4-ALK fusion and aberrant ALK expression. Overall mutation frequencies of EGFR and EML4-ALK are $\sim 25 \%$ and $1-7 \%(3,6)$, respectively, which suggests that $>65 \%$ of lung cancer patients lack specific molecular targets for treatment. Identification of new targets either for diagnosis or therapy is important in the effort to reduce mortality by lung cancer.

NKX2-1 (TTF1 or TITF1 or thyroid transcription factor-1) was originally identified in thyroid and lung (8). Many efforts have been made to identify characteristics of $N K X 2-1$ not only in thyroid but also in other organs, including the lung. NKX2-1 was first thought to act as a lung oncogene $(9,10)$, but later study suggested that it may act as a tumor suppressor in lung adenocarcinoma (11). Notwithstanding the clear contribution of NKX2-1 to lung development and homeostasis, it is not clear how to inhibit or boost NKX2-1 for the treatment of lung cancer. This gene is not clearly amplified, overexpressed, or mutated and as such no clear agonistic or antagonistic molecular targets of NKX2-1 have been developed for lung cancer.

In order to find potential lung cancer or general thoracic malignancy-related markers associated with NKX2-1, we screened other binding partners of NKX2-1, filtering out known type II pneumocyte and clara cell markers. One of the interesting binding partners of NKX2-1 is TSHR (12). NKX2-1 binds the promoter of TSHR and promotes constitutive TSHR expression and TSH/cAMP-induced negative regulation of TSHR $(12,13)$. Activating and inactivating mutations have 
Table I. Past medical and surgical history of case 611 with TSHR mutation.

History

Coronary artery disease, with history of myocardial infarction,

status post percutaneous coronary intervention with stent placement

Chronic obstructive pulmonary disease (COPD)

Bilateral severe carotid stenosis, status post bilateral carotid endarterectomies

Peripheral vascular disease

Hypertension

Hyperlipidemia

Hiatal hernia

Gastroesophageal reflux disease

Diverticulosis

Primary hyperparathyroidism, status post parathyroidectomy

Nephrolithiasis, status post bilateral nephrolithotomies

Pertussis, causing right pneumothorax, status post right thoracotomy

Bilateral cataracts, status post bilateral cataract repair

been reported in many thyroid-related diseases, such as hyper/ hypothyroidism and thyroid cancer $(14,15)$. Most activating mutations are known to be associated with autoimmune diseases of the thyroid $(14,15)$. Epigenetic changes in TSHR have been reported to cause a significant down-regulation and decreased expression of TSHR in thyroid cancer (16). TSHR has not been studied in lung cancer or thoracic malignancies except in a mutation screening of a lung metastasis from thyroid cancer (17). No TSHR mutation was found in the pulmonary metastasis of thyroid cancer (17). As such, to date it is not clear whether TSHR is genetically involved in primary lung cancer development. Thus, we screened for TSHR mutation, DNA copy number, and mRNA expression in 96 lung adenocarcinoma and matched normal tissues. In addition to lung adenocarcinoma, other thoracic malignancies including squamous cell carcinoma (SCC) and malignant pleural mesothelioma (MPM) were measured for TSHR protein expression by immunohistochemistry.

\section{Materials and methods}

Patient samples. The Committee on Human Research (CHR) of UCSF reviewed and approved the application for the collection of tissue samples from patients with thoracic malignancies (approval number: 10-03352). All samples were collected under the IRB approval and written informed consents were obtained from all patients in this study.

TSHR mutation screening. Ninety-six lung adenocarcinoma tissue samples screened for our previous study (18) were used for TSHR mutation screening. All coding regions of TSHR were screened using PCR primers as previously described (19). Amplification was performed at $58-62^{\circ} \mathrm{C}$ using $20 \mathrm{ng}$ of genomic DNA in 96 sample pairs, 10X PCR buffer supplemented with $1.5 \mathrm{mM} \mathrm{MgCl}_{2}, 10 \mathrm{pmol}$ of each primer, $50 \mathrm{mM}$ of each dNTP, and 0.2 units of Taq polymerase (Qiagen) in a total reaction volume of $25 \mu \mathrm{l}$. The PCR products were cleaned up using $1 \mu \mathrm{l}$ of SAP $(1 \mathrm{U} / \mu \mathrm{l}), 0.1 \mu \mathrm{l}$ of Exol enzyme $(10 \mathrm{U} / \mu \mathrm{l}), 1 \mu \mathrm{l}$ of 1X PCR buffer, $2.4 \mu \mathrm{l}$ of 10X NEB buffer (type 3), $5 \mu \mathrm{l}$ of the PCR product, and $15 \mu \mathrm{l}$ of water. Bi-directional sequencing was performed using the Taq dideoxy-terminator cycle sequencing kit and an ABI 3730xl DNA analyzer (Applied Biosystems, Foster City, CA, USA).

TSHR mRNA expression analysis in 96 lung adenocarcinoma and matched normal tissues. RNA from 96 snap-frozen lung adenocarcinoma and matched normal tissues was extracted with TRIzol (Invitrogen), treated with DNase I (New England Biolabs), and purified with the RNeasy RNA purification kit (Qiagen). cDNA was synthesized using a reverse transcript RT III (Invitrogen). TSHR expression was quantified by Taqman real-time PCR (ABI, 7900HT) using 96 lung adenocarcinoma and matched normal tissues in triplicate (TSHR expression probe: 4331182, ABI). GAPDH was selected to normalize cDNA input. $\triangle \mathrm{CT} 2$ was calculated from an expression difference between $T S H R$ and GAPDH. $\triangle \mathrm{CT}$ values were then used to compare $T S H R$ expression between tumor and normal samples.

TSHR DNA copy number analysis in 96 lung adenocarcinoma and matched normal tissues. DNA copy number analysis of TSHR was done in the same 96 pairs of samples (TSHR DNA probe: Hs01214599_cn). DNA copy number assay for TSHR was performed using Taqman real-time PCR. All samples were analyzed in triplicate and RNase $\mathrm{P}$ gene (VIC/Tamra probe) was used for internal control to normalize DNA quantity. Taqman probes were labeled by FAM/non-fluorescent quencher. Sample DNA (10 ng) were analyzed on a 384-well plate using $2 \mathrm{X}$ Taqman Universal PCR master mix with no AmpErase UNG. Deletion or gain was determined by $\Delta \mathrm{CT}$ calculation and analyzed by software provided by ABI. Further data analysis was also performed manually. After average $\Delta \mathrm{CT}$ values of normal controls were calculated, each $\Delta \mathrm{CT}$ value of samples 
$\mathbf{A}$
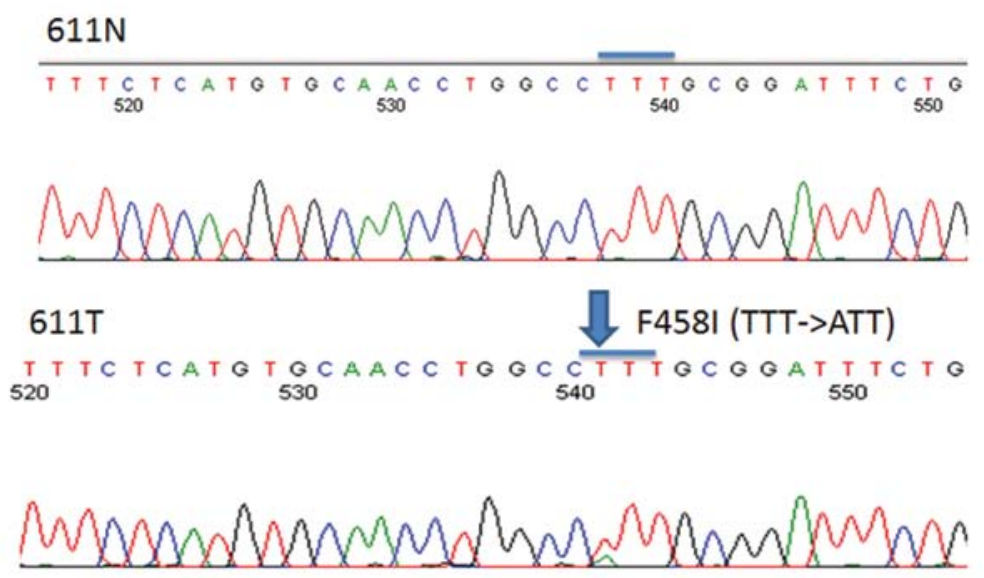

B

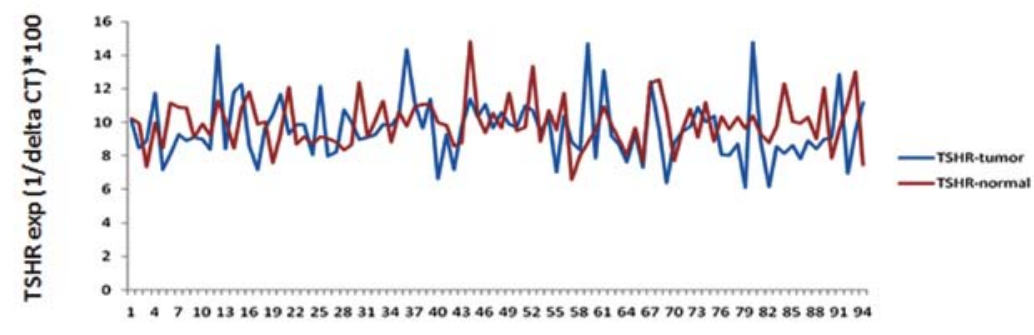

C

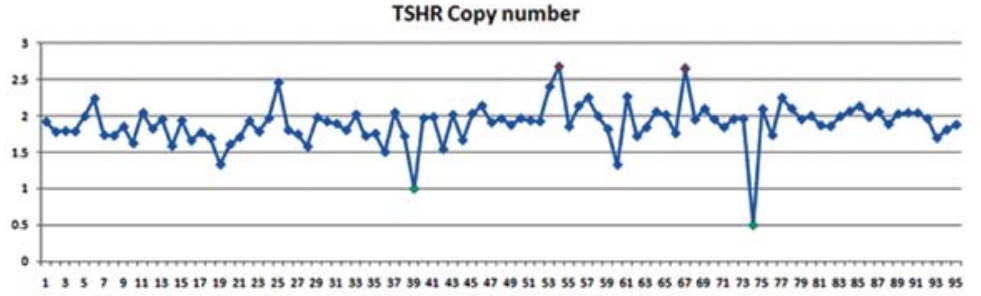

Figure 1. Somatic mutation of TSHR in lung adenocarcinoma patient (ID: 611) and TSHR mRNA expression and DNA copy number in lung adenocarcinoma and matched normal tissues. A missense mutation of TSHR at exon 10 (codon 458) was found in a primary lung cancer sample and not its matched normal tissue. The mutation is located at the transmembrane domain where most TSHR mutations have been reported in hyper/hypothyroidism, thyroid cancer and other thyroid-related diseases. TSHR expression was checked by Taqman using 96 lung adenocarcinoma and matched normal tissues (TSHR expression probe: 4331182, ABI). There was no statistically significant mRNA expression change between normal and matched tumor tissues (paired t-test $\mathrm{p}=0.118)$. $\mathrm{Y}$ axis: TSHR expression [(1/ACT)*100] (A) DNA copy number analysis of TSHR was done in the same 96 pairs of samples (TSHR DNA probe: Hs01214599_cn) (B) Deletion or gain was determined by $\Delta \mathrm{CT}$ calculation and analyzed by software provided by ABI. Two potential amplifications (red) and two deletions (green) were identified in $96 \mathrm{AD}$ samples. Most of the samples except these four showed normal ranges of DNA copy number, which means LOH or amplification are not major genetic events of TSHR contributing lung tumor development.

was divided by this normal value. $\Delta \Delta \mathrm{CT}$ values were then calculated and the threshold level for copy number was set as $<1.2$ for deletion and $>2.6$ for gain.

Immunohistochemistry (IHC). Ten pairs of matched normal and lung adenocarcinoma (AD) and squamous cell carcinoma (SCC), and five malignant pleural mesothelioma (MPM) samples were stained with anti-TSHR antibody (ab5492, Abcam). Sections $(5 \mu \mathrm{m})$ were hydrated in xylene and graded concentrations of ethanol, then steamed in citrate (Biogenex) for $20 \mathrm{~min}$. Slides were then incubated at room temperature with a blocking solution (10\% goat serum in TBS and $1 \%$ BSA) for $1 \mathrm{~h}$. Blocking solution was then removed and the primary antibody was applied. The slides were incubated at $4^{\circ} \mathrm{C}$ overnight. The slides were incubated using reagents from the Invitrogen Histostain Plus Broad Spectrum kit (85-9643) according to the protocol provided by Invitrogen.

\section{Results}

Somatic TSHR mutation. Out of 96 lung adenocarcinoma samples, one mutation was identified in adenocarcinoma tissue (patient ID: 611) that did not appear in matched normal tissue (Fig. 1A). A missense mutation at exon 10 (codon 458, TTT $\rightarrow$ ATT, F458I) was identified at the transmembrane domain of TSHR (Fig. 1A). The results suggest an overall observed $T S H R$ mutation frequency of $1 \%$ (one out of 96 AD). We investigated the prior clinical history of the TSHR-mutated patient (patient ID: 611) to identify unique clinical insight. The patient was a 67-year-old (at the time of her second operation, during 
A

TSHR

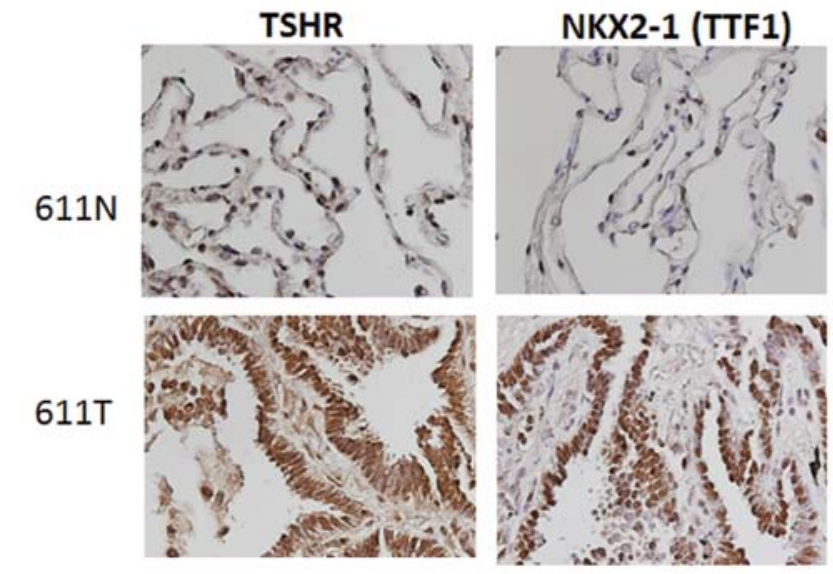

B

$\mathrm{N}$
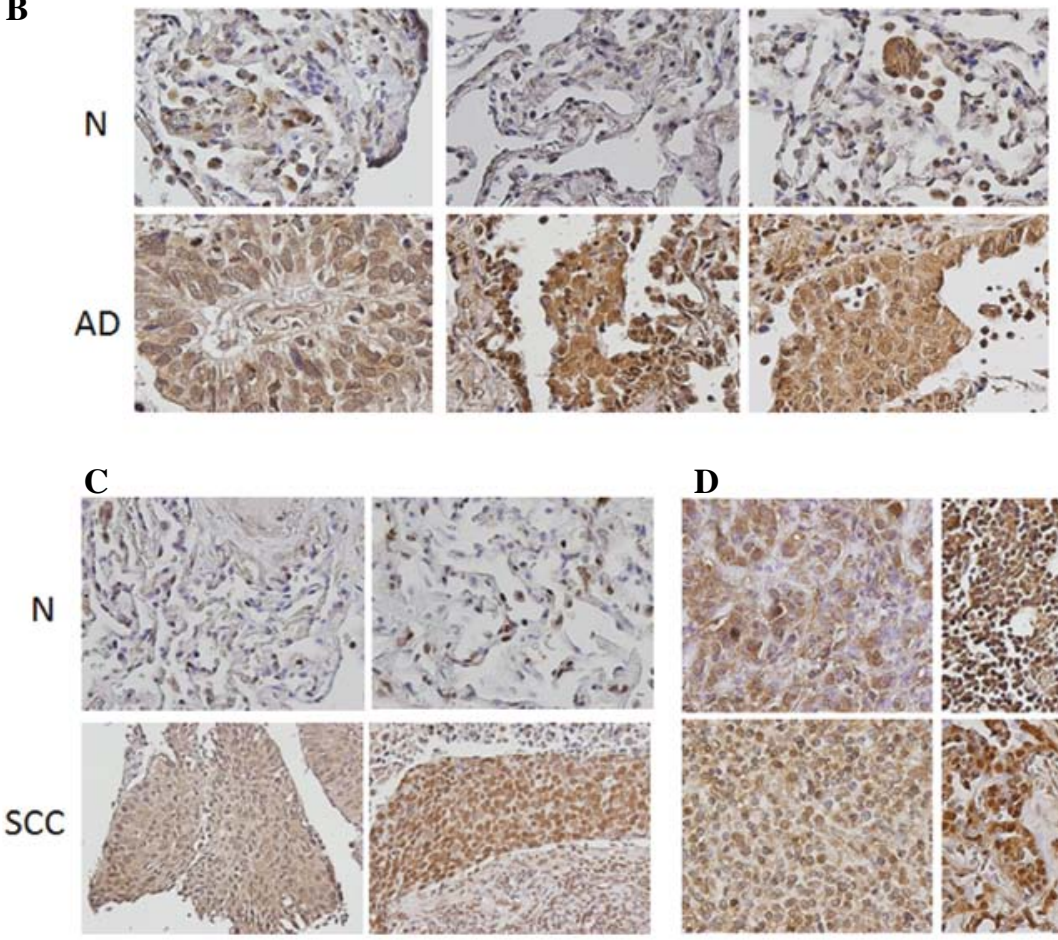

D

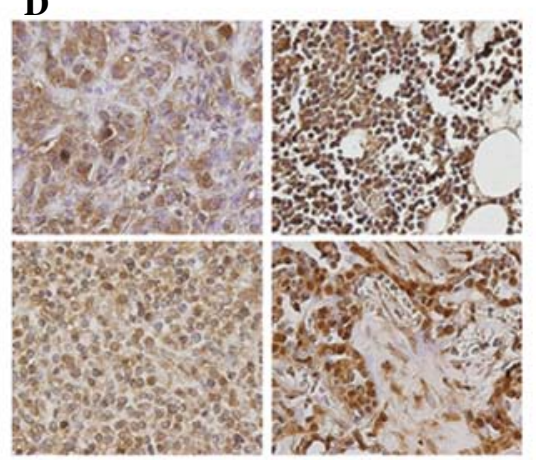

Figure 2. Immunohistochemistry of TSHR in lung adenocarcinoma (AD), squamous cell carcinoma (SCC), and malignant pleural mesothelioma (MPM). Overexpression of TSHR and NKX2-1 (TTF1) in tumor samples compared to matched normal tissues in patient 611 with TSHR somatic mutation (A). Representative examples of three pairs of matched normal and lung AD (B), two pairs of matched SCC (C), and four MPM (D) specimens were stained with anti-TSHR antibody (ab5492, Abcam). High TSHR expression was detected in tumor specimens, but only mild in the matched normal specimens (B-D). MPM specimens were also analyzed for TSHR protein level and high TSHR levels compatible with those of AD and SCC were detected (C). Microscope magnification: $\mathrm{x} 20, \mathrm{x} 40$.

which the specimen was collected) Caucasian woman with a history of coronary artery disease, carotid stenosis, chronic obstructive pulmonary disease (COPD) and a 50-pack-year smoking history who had presented 15 months earlier with a 3-year history of cough and 6-month history of hoarseness. CT scan showed bilateral upper lobe lung lesions, and fine needle aspiration suggested bilateral lung adenocarcinoma. Her case was discussed at our institution's thoracic oncology tumor board, and the lesions were thought likely to be two primary tumors. She first underwent left upper lobectomy, and pathology showed stage IIB moderately differentiated adenocarcinoma. Postoperatively, she received carboplatin and gemcitabine for 2 cycles, then carboplatin and paclitaxel for 4 cycles. Imaging revealed stable disease, so she then under- went right upper lobectomy, during which time our tumor and adjacent lung specimens were collected. Pathology showed stage IB adenocarcinoma with features of bronchioloalveolar carcinoma. This patient had no signs or symptoms associated with thyroid cancer or diseases (Table I). A systematic review of English-language manuscripts available in the PubMed database yielded no prior report that TSHR is associated with COPD or coronary artery disease.

TSHR mRNA expression and copy number. To determine if $T S H R$ is amplified or overexpressed in lung AD samples, we measured TSHR gene expression in $96 \mathrm{AD}$ and matched normal tissues using a real-time quantitative PCR (Fig. 1B). There was no clear up-regulation of TSHR in tumor samples 
compared to the matched normal tissues. TSHR DNA copy number was then checked, and only two potential amplifications of TSHR in 96 AD samples were found (Fig. 1C), which suggests that TSHR amplification might not be a major genetic event in lung cancer. For deletion, only additional two samples showed clear deletions in 96 AD samples.

TSHR protein expression by immunohistochemistry. Next, we checked TSHR protein expression in tumor and matched normal samples of the patient harboring the TSHR mutation. Both TSHR and NKX2-1 (TTF1) were generally overexpressed in tumor samples compared to normal tissues (Fig. 2A). Additional samples of three different subtypes of thoracic malignancies, adenocarcinoma (AD), squamous cell carcinoma (SCC), and malignant pleural mesothelioma (MPM), were analyzed for TSHR expression by immunohistochemistry (IHC) (Fig. 2B-D). High TSHR protein expression was found in AD and SCC while low or mild expression was detected in matched normal samples (Fig. 2B and C). For MPM, only tumor samples were stained for TSHR and high TSHR expression was detected (Fig. 2D). Although overall mRNA expression of $T S H R$ was low and not significantly different in normal and tumor tissues (Fig. 1B), the protein level of TSHR is meaningfully high in all three subtypes of thoracic malignancies, AD (Fig. 2B), SCC (C) and MPM (D).

\section{Discussion}

Lung cancer is the leading cause of cancer death in men and women in the US and world-wide (1). Five-year survival has stubbornly remained at a dismal $15-17 \%$ for a decade, making it one of the most lethal cancers. Chronic respiratory diseases are another major cause of deaths. Worldwide, COPD (Chronic Obstructive Pulmonary Disease) is responsible for 3 million deaths annually $(20,21)$. COPD is relatively irreversible and the rate of incidence is increasing, such that COPD is expected to be the 3rd or 4th leading cause of death by $2030(20,21)$ as a result of increases in smoking and air pollution. However, as only $15-20 \%$ of smokers are estimated to develop either lung cancer or COPD, there must exist additional major genetic mechanisms driving the development of these diseases (22-24). Up to half of lung cancer patients suffer from COPD and $1 \%$ of COPD patients develop lung cancer per year $(24,25)$. The prognosis of lung cancer patients with COPD is worse than that of patients without COPD (24). Although several genetic and epigenetic mechanisms have been proposed as playing a role in lung cancer development in COPD patients, (22-26) the exact mechanisms by which some patients with COPD later develop lung cancer remains unclear.

We screened for mutations in TSHR based on its putative relationship with NKX2-1, a potential tumor suppressor and type II pneumocyte marker in lung (11). The mutation frequency of $T S H R$ in lung cancer in our study is $\sim 1 \%$, similar to EML4-ALK, a fusion protein estimated to play a role in 1-7\% of lung adenocarcinoma $(3,5)$. When analyzing clinical characteristic of patient 611 with TSHR mutation, we found that this patient had a complicated prior medical history significant for carotid stenosis, coronary artery disease and COPD. It was reported that COPD is frequent in higher grade and non-BAC adenocarcinoma $(24,27)$. Thus, it is possible that THSR may be involved in the development of a BAC-type lung adenocarcinoma arising from a patient population with pre-existing COPD, although further systematic study with large patient number will be required to validate this.

$T S H R$ has been reported to be mutated in many thyroidrelated diseases (14). Activating TSHR mutations have been reported in congenital hyperthyroidism, toxic multinodular goiter and hot nodules. Inactivating TSHR mutations have been reported in congenital hypothyroidism, multinodular goiter and cold nodules (14). Although several studies have reported TSHR somatic mutations in thyroid cancer (15), epigenetic changes (methylation) mainly seem to cause reduced expression of TSHR in thyroid cancer $(14,16)$. The novel mutation identified in this study is located at codon 458 (exon 10), close to a proven functional M453T TSHR transmembrane domain mutation, where most activating $T S H R$ mutations occur $(14,15)$. It was reported that an activating germline M453T TSHR mutation caused non-autoimmune hyperthyroidism $(28,29)$.

We hypothesized that the identified TSHR mutation at the transmembrane domain would cause an overexpression of TSHR or its binding partner, NKX2-1. We quantified TSHR mRNA levels in both lung adenocarcinoma and matched normal tissues and did not find a statistically significant change (Fig. 1B). At the DNA level, two samples showed potential copy number amplifications while two other samples showed clear deletions (Fig. 1C). Next, we checked TSHR protein level by immunohistochemistry. A significant overexpression of both TSHR and NKX2-1 in patient 611's tumor samples was observed (Fig. 2A). We also checked other subtypes of thoracic malignancies, AD, SCC and MPM, for TSHR protein level. As shown in Fig. 2B-D, high TSHR expression was identified in tumor samples but not in the matched normal tissues. This indicates that TSHR protein expression, not mRNA expression, may potentially be a useful lung tumor marker for either diagnostic or therapeutic purposes. An anti-TSHR antibody-based ELISA assay performed on blood specimens may provide a simple and reliable method for earlier detection of lung cancer.

Taken together, we first identified a somatic mutation of TSHR in a patient presenting with lung adenocarcinoma with BAC features and a prior medical history significant for COPD and coronary artery disease. Our observed mutation frequency of $T S H R$ is $\sim 1 \%(1 / 96)$ in lung adenocarcinoma patients, which suggests that mutation of TSHR is not a major event in lung carcinogenesis generally. It does not appear that TSHR contributes to lung cancer development by classical genetic events such as mutation, amplification, or overexpression. When considering a significant overexpression of TSHR in tumor compared to matched normal samples, TSHR protein, not DNA or mRNA, may be used for either diagnostic or therapeutic purpose. Although further studies with larger numbers of samples are required, it appears possible that a subgroup of lung adenocarcinoma patients, such as those with BAC, COPD and/or cardiovascular diseases, may be susceptible to a TSHR mutation.

\section{Acknowledgements}

This work was supported by the Barbara Isackson Lung Cancer Research Fund, The Eileen D. Ludwig Endowed Fund, and Kazan, McClain, Abrams, Fernandez, Lyons, Greenwood, Harley \& Oberman Foundation Fund for Thoracic Oncology Research. I.J.K. is supported by the UALC (Uniting Against Lung Cancer) foundation. 


\section{References}

1. Edwards BK, Ward E, Kohler BA, et al: Annual report to the nation on the status of cancer, 1975-2006, featuring colorectal cancer trends and impact of interventions (risk factors, screening, and treatment) to reduce future rates. Cancer 116: 544-573, 2010

2. Lynch TJ, Bell DW, Sordella R, et al: Activating mutations in the epidermal growth factor receptor underlying responsiveness of non-small-cell lung cancer to gefitinib. N Engl J Med 350: 2129-2139, 2004.

3. Soda M, Choi YL, Enomoto M, et al: Identification of the transforming EML4-ALK fusion gene in non-small-cell lung cancer. Nature 448: 561-566, 2007.

4. Dahabreh IJ, Linardou H, Siannis F, et al: Somatic EGFR mutation and gene copy gain as predictive biomarkers for response to tyrosine kinase inhibitors in non-small cell lung cancer. Clin Cancer Res 16: 291-303, 2010.

5. Pao W, Miller VA, Politi KA, et al: Acquired resistance of lung adenocarcinomas to gefitinib or erlotinib is associated with a second mutation in the EGFR kinase domain. PLoS Med 2: e73, 2005.

6. Horn L and Pao W: EML4-ALK: honing in on a new target in non-small-cell lung cancer. J Clin Oncol 27: 4232-4235, 2009.

7. Koivunen JP, Mermel C, Zejnullahu K, et al: EML4-ALK fusion gene and efficacy of an ALK kinase inhibitor in lung cancer. Clin Cancer Res 14: 4275-4283, 2008.

8. Guazzi S, Price M, De Felice M, et al: Thyroid nuclear factor 1 (TTF-1) contains a homeodomain and displays a novel DNA binding specificity. EMBO J 9: 3631-3639, 1990

9. Weir BA, Woo MS, Getz G, et al: Characterizing the cancer genome in lung adenocarcinoma. Nature 450: 893-898, 2007.

10. Kwei KA, Kim YH, Girard L, et al: Genomic profiling identifies TITF1 as a lineage-specific oncogene amplified in lung cancer. Oncogene 27: 3635-3640, 2008.

11. Winslow MM, Dayton TL, Verhaak RG, et al: Suppression of lung adenocarcinoma progression by Nkx2-1. Nature 473 : 101-104, 2011.

12. Civitareale D, Castelli MP, Falasca P, et al: Thyroid transcription factor 1 activates the promoter of the thyrotropin receptor gene. Mol Endocrinol 7: 1589-1595, 1993.

13. Shimura H, Shimura Y, Ohmori M, et al: Single strand DNA-binding proteins and thyroid transcription factor-1 conjointly regulate thyrotropin receptor gene expression. Mol Endocrinol 9: 527-539, 1995

14. Davies TF, Yin X and Latif R: The genetics of the thyroid stimulating hormone receptor: history and relevance. Thyroid 20 727-736, 2010

15. Hébrant A, van Staveren WC, Maenhaut C, et al: Genetic hyperthyroidism: hyperthyroidism due to activating TSHR mutations. Eur J Endocrinol 164: 1-9, 2011.
16. Xing M, Usadel $\mathrm{H}$, Cohen $\mathrm{Y}$, et al: Methylation of the thyroidstimulating hormone receptor gene in epithelial thyroid tumors: a marker of malignancy and a cause of gene silencing. Cancer Res 63: 2316-2321, 2003.

17. Führer D, Tannapfel A, Sabri O, et al: Two somatic TSH receptor mutations in a patient with toxic metastasising follicular thyroid carcinoma and non-functional lung metastases. Endocr Relat Cancer 10: 591-600, 2003.

18. Choi H, Kratz J, Pham P, et al: Development of a practical fast mutation screening assay for human lung adenocarcinoma. Int $J$ Oncol 40: 1900-1906, 2012.

19. Narumi S, Muroya K, Abe Y, et al: TSHR mutations as a cause of congenital hypothyroidism in Japan: a population-based genetic epidemiology study. J Clin Endocrinol Metab 94: 1317-1323, 2009.

20. Stevenson CS and Birrell MA: Moving towards a new generation of animal models for asthma and COPD with improved clinical relevance. Pharmacol Ther 130: 93-105, 2011.

21. Whitsett J, Wert S and Weaver T: Alveolar surfactant homeostasis and the pathogenesis of pulmonary disease. Annu Rev Med 61: 105-119, 2010.

22. Spitz MR, Wei Q, Dong Q, et al: Genetic susceptibility to lung cancer: the role of DNA damage and repair. Cancer Epidemiol Biomarkers Prev 12: 689-698, 2003.

23. Cohen BH: Chronic obstructive pulmonary disease: a challenge in genetic epidemiology. Am J Epidemiol 112: 274-288, 1980.

24. Sekine Y, Katsura H, Koh E, et al: Early detection of COPD is important for lung cancer surveillance. Eur Respir J: Nov 16, 2011 (Epub ahead of print).

25. Skillrud DM, Offord KP and Miller RD: Higher risk of lung cancer in chronic obstructive pulmonary disease. A prospective matched, controlled study. Ann Intern Med 105: 503-507, 1986.

26. Wright JG and Christman JW: The role of nuclear factor kappaB in the pathogenesis of pulmonary diseases: implications for therapy. Am J Respir Med 2: 211-219, 2003.

27. Brody JS and Spira A: State of the art. Chronic obstructive pulmonary disease, inflammation, and lung cancer. Proc Am Thorac Soc 3: 535-537, 2006.

28. Kraemer S, Rothe K, Pfaeffle R, et al: Activating TSH-receptor mutation (Met453Thr) as a cause of adenomatous non-autoimmune hyperthyroidism in a 3-year-old boy. J Pediatr Endocrinol Metab 22: 269-274, 2009.

29. Supornsilchai V, Sahakitrungruang T, Wongjitrat N, et al: Expanding clinical spectrum of non-autoimmune hyperthyroidism due to an activating germline mutation, p.M453T, in the thyrotropin receptor gene. Clin Endocrinol 70: 623-628, 2009. 